\title{
Transición hacia la TDT y panorama actual de la televisión local en la Comunidad Autónoma Vasca
}

\author{
Leire Gómez RuBio \\ Universidad del País Vasco \\ leiregom@yahoo.es
}

Recibido: $04 / 10 / 2012$

Aceptado: 23/01/2013

\section{Resumen}

La digitalización y la crisis económica han hecho que, hoy, la situación de la televisión local en la Comunidad Autónoma Vasca difiera de la que el sector presentaba hace unos años, tanto en el número de emisoras que se mantienen en activo, como en la actividad que éstas desarrollan y los medios de los que se sirven, al tiempo que los grupos de comunicación han materializado, con diferentes actuaciones, su pérdida de interés por seguir presentes en el mercado audiovisual local vasco. El resultado de todo ello ha sido la convivencia, durante los últimos años, de los cierres de emisoras con una trayectoria importante en la televisión local vasca y la apuesta de otras por emitir a través de la Red.

Palabras clave: televisión local, Euskadi, TDT local, audiovisual vasco

\section{The Transition to DTT and Current Outlook to Local Television Broadcasters Panorama in the Basque Country}

\begin{abstract}
Recent digitalization, as well as the global economic crisis, have make become a whole new situation for the local TV on Basque Country, both in the broadcasting station amount and on the activity they usually develop and the way they do it. At the same time, great communication media have finally displayed their lack of interest on the local audiovisual market. As a result, on last times the closures and shutdown of many broadcasting stations, even of those with a longer trajectory on the development of local audiovisual, live together with new experiences of net or web broadcasting.

Keywords: local television, Euskadi, Basque Country, local DTT, basque audiovisual

Referencia normalizada

GÓMEZ RUBIO, Leire (2013): “Transición hacia la TDT y panorama actual de la televisión local en la Comunidad Autónoma Vasca”. Estudios sobre el Mensaje Periodístico. Vol. 19, Núm. especial marzo, págs.: 243-251. Madrid, Servicio de Publicaciones de la Universidad Complutense.
\end{abstract}

Sumario: 1. Introducción. 2. Objetivos y metodología. 3. La televisión local en Euskadi en los albores de la era digital; 3.1. Primeras consecuencias de la digitalización. 4. Panorama actual de la televisión local vasca. 5. La oportunidad de Internet. 6. Conclusiones. 7. Referencias bibliográficas.

\section{Introducción}

Aumento del número de número de canales de televisión, mejor gestión del espectro radioeléctrico, abaratamiento de los costes de transmisión, televisión personal e interactiva, programaciones temáticas y generalistas y confluencia de servicios añadidos, sobre todo derivados de la informática y de la telefonía móvil, son algunos de los nuevos usos y posibilidades de la TDT. Nuevos servicio, en definitiva, que apuntaban hacia una revolución sin precedentes en el sector audiovisual en general. En el caso de la televisión local, además, la era digital suponía la oportunidad, por primera vez, de configurarse "de manera legal y para el medio-largo plazo, después de un largo tiempo de indefinición, regulación insuficiente y desregulación oculta" (Badillo y Ortega, 2008:web). 
No obstante, más de dos años después del definitivo apagón analógico, en abril de 2010, la digitalización, unida a la crisis económica y a la falta de apoyo financiero por parte de las administraciones públicas, ha puesto de manifiesto el fracaso de la TDT local en la Comunidad Autónoma Vasca. De hecho, actualmente Euskadi cuenta con el menor número de televisiones locales ${ }^{1}$ desde que, en 2007, el Gobierno Vasco procediera al reparto de canales digitales. Los ceses de emisoras con una importante trayectoria dentro del audiovisual local vasco y la escasa puesta en marcha de nuevas iniciativas entre las concesionarias son dos de las características que han guiado la transición hacia la TDT local en Euskadi.

Junto a esto, otras emisoras han buscado nuevas vías de negocio, encontrado en Internet el garante a la continuidad de sus emisiones, además de abrirse ante ellas las nuevas posibilidades que la combinación del ordenador, la Red y la televisión suponen, "como chatear mientras se ve un canal, capacidad de valorar las emisiones televisivas, crear listas de reproducción o descubrir nuevos programas a través de personas con gustos similares (Pérez y Santos, 2009: web)

\section{Objetivos y metodología}

El objetivo de este artículo es presentar cómo ha tenido lugar la transición hacia la tecnología digital de la televisión local en Euskadi desde que, en el año 2007, el Gobierno Vasco hiciera público el reparto de licencias digitales, así como exponer la situación actual de la televisión local en la Comunidad Autónoma Vasca. Para ello, en las siguientes líneas se muestra un análisis comparativo de la realidad del sector en 2007 y en 2012, atendiendo al número de emisoras en activo, su distribución y localización geográfica en función de las demarcaciones contempladas por el Plan Técnico Nacional de la Televisión Digital Local, titularidad, vinculación con grupos de comunicación y asociaciones de televisión local y uso que las emisoras realizan de la Red.

La hipótesis de partida es que, en el caso de Euskadi, la digitalización, unida a la crisis económica y, por ende, publicitaria, se ha convertido en una amenaza que ha terminado con el optimismo inicial, en el que la nueva tecnología ni tan siquiera ha asegurado la viabilidad de la televisión local "gracias al desarrollo que puede suponer para la industria televisiva el crecimiento del interés por los contenidos y la información local, y la posibilidad de explotar un nuevo campo para la publicidad" (García, 2005: web).

La primera parte del trabajo de campo se realizó en el último trimestre de 2007, con el propósito de conocer cómo afrontaban la digitalización las televisiones concesionarias de licencia digital ${ }^{2}$. En este punto, se diseñó y envío a cada una de las televi-

1 Se considera televisión local a aquellas estaciones que realizan y emiten algún espacio de producción propia. Quedan excluidas de tal consideración aquellas que, aun disponiendo de la correspondiente licencia digital, actúan como meros repetidores de la programación en cadena de algún grupo de comunicación.

2 El resultado de este trabajo fue la tesis doctoral "Análisis prospectivo de un modelo de proximidad: la televisión local en la Comunidad Autónoma Vasca ante la llegada de la TDT”, defendida por la autora de este artículo en enero de 2011 en la Universidad del País Vasco- 
siones objeto de estudio un cuestionario abierto compuesto por seis fichas con más de cien entradas, con el que se procedió a la recogida de datos referentes a la identificación de cada una de las emisoras, a su descripción técnica y humana, a sus emisiones, financiación, publicidad y a sus programaciones semanales. Una vez recopilados y analizados estos datos, se realizaron 17 entrevistas en profundidad a los responsables de las emisoras que habían resultado adjudicatarias de un canal digital local, excluyéndose de este análisis a aquellas televisiones que realizaban sus emisiones únicamente a través de la Red.

Los datos obtenidos en esta primera fase del estudio han sido actualizados durante los meses de julio y septiembre de 2012 mediante consultas periódicas realizadas a los responsables y trabajadores de las televisiones locales que han continuado con su actividad tras el encendido digital, así como a través de la consulta de las fuentes hemerográficas localizadas al respecto.

\section{La televisión local en Euskadi en los albores de la era digital}

Cuando el Gobierno Vasco procede, en julio de 2007, al reparto de licencias de canales digitales locales, son un total de 25 las televisiones locales que, en ese momento, desarrollan su actividad en Euskadi: 14 en Gipuzkoa, 8 en Bizkaia y 3 en Araba, distribuida en 16 localidades, de modo que son el 6\% de los municipios de la Comunidad Autónoma los que cuentan, al menos físicamente, con una emisora de este tipo. Se trata de canales ubicados de forma mayoritaria en dos tipos de poblaciones en función del número de habitantes: aquellos con más de 200.000 habitantes, con el $28 \%$ de las televisiones locales, entre las que se encuentran dos de las tres capitales de provincia, Bilbao y Vitoria-Gasteiz; y aquellos con menos de 20.000 habitantes, también con el $28 \%$.

Desde una perspectiva administrativa, la digitalización permitía que esta modalidad de televisión en Euskadi creciera en casi un 60\%, ya que el Plan Técnico Nacional de Televisión Digital Local divide la Comunidad Autónoma Vasca en 15 demarcaciones, a cada una de las cuales les asigna un múltiplex con capacidad para cuatro canales de televisión local. Se trata de demarcaciones, además, que cuentan con un carácter supramunicipal y en las que, a excepción de dos -Llodio y Mungia-, ya existe una actividad televisiva local en alguna de las localidades incluidas dentro de la misma. No obstante, el reparto del Plan Técnico Nacional hace que las posibilidades de desarrollo de la TDT local sean escasas en algunas demarcaciones, como son los casos de las de Donostia, Bilbao y Gasteiz: la primera de ellas, en esos momentos, cuenta con cinco televisiones locales, de las que cuatro transmiten sus emi-

Euskal Herriko Unibertsitatea. La investigación abordó el estudio de las televisiones locales en activo en las tres provincias de la Comunidad Autónoma Vasca durante el año 2007 que fueron beneficiarias de una licencia de TDT local. Se hizo especial hincapié en su área de cobertura, organización técnica y empresarial, relación con grupos de comunicación y asociaciones de televisión local, financiación, audiencias, programación e idioma de las emisiones, además de dar respuesta a los nuevos retos y posibilidades que la tecnología digital brinda a este tipo de emisoras. 
siones a través de ondas hertzianas y el cable y una a través de Internet; Bilbao cuenta con cuatro estaciones locales, de las que también tres se difunden a través de ondas hertzianas y cable y una por al Red, y la capital alavesa cuenta con tres estaciones, las tres por ondas hertzianas y cable.

Tabla 1. Demarcaciones establecidas por el Plan Técnico Nacional de Televisión Digital Local y televisiones locales en activo en la Comunidad Autónoma Vasca en 2007 (elaboración propia)

\begin{tabular}{|l|l|c|}
\hline DEMARCACIONES & MUNICIPIOS & $\begin{array}{c}\text { TVLs en } \\
\text { activo en } \\
\mathbf{2 0 0 7}\end{array}$ \\
\hline Llodio & (2) Llodio, Amurrio & 0 \\
\hline Vitoria-Gasteiz & (1)Vitoria-Gasteiz & 3 \\
\hline Beasain & (7) Beasain, Zumarraga, Ordizia, Legazpi, Urretxu, Ataun, Ormaiztegi & 2 \\
\hline Eibar & (4) Eibar, Ermua, Elgoibar, Soraluze & 1 \\
\hline Arrasate-Mondragón & (5) Arrasate-Mondragón, Bergara, Oñati, Aretxabaleta, Eskoriatza & 2 \\
\hline Donostia-San Sebastián & $\begin{array}{l}\text { (7) Donostia, Hernani, Lasarte-Oria, Andoain, Urnieta, Usurbil, } \\
\text { Astigarraga }\end{array}$ & 5 \\
\hline Tolosa & (4) Tolosa, Villabona, Anoeta, Itsasondo & 1 \\
\hline Zarautz & (5) Zarautz, Azpeitia, Azkoitia, Zumaia, Orio & 2 \\
\hline Irun & (6) Irun, Renteria, Pasaia, Hondarribia, Oiartzun, Lezo & 1 \\
\hline Barakaldo & $\begin{array}{l}\text { (8) Barakaldo, Portugalete, Santurtzi, Sestao, Trapagaran, Abanto- } \\
\text { Zierbana, Ortuella, Muskiz }\end{array}$ & 1 \\
\hline Bermeo & (5) Bermeo, Gernika-Lumo, Ondarroa, Lekeitio, Markina-Xemein & 1 \\
\hline Bilbao & (5) Bilbao, Basauri, Galdakao, Arrigorriaga, Etxebarri & 4 \\
\hline Durango & (5) Durango, Amorebieta-Etxano, Elorrio, Abadiño, Berriz & 1 \\
\hline Mungia & $\begin{array}{l}\text { (15) Mungia, Derio, Sondika, Zamudio, Lezama, Loiu, Bakio, } \\
\text { Larrabetzu, Gatika, Gamiz-Fika, Laukiz, Maruri-Jatabe, Meñaka, } \\
\text { Arrieta, Fruiz }\end{array}$ & 0 \\
\hline Getxo & $\begin{array}{l}\text { (9) Getxo, Leioa, Erandio, Sopela, Berango, Plentzia, Urdulliz, Barrika, } \\
\text { Lemoiz }\end{array}$ & 1 \\
\hline & \multicolumn{1}{|c|}{ 88 } & 25 \\
\hline
\end{tabular}

De las 25 emisoras en activo, 19 son las que resultan beneficiarias de un canal digital por el Ejecutivo de Lakua. Una de las principales características que se desprende del reparto es que una amplia mayoría de las empresas adjudicatarias están vinculadas con grupos de comunicación y asociaciones de televisión local -Prisa, Vocento, Local Media y Hamaika Telebista Hedatzeko Taldea-. Ello les hace poseedores de unas mayores posibilidades técnicas y económicas, respondiendo así a uno de los criterios tenidos en cuenta por el Gobierno Vasco a la hora de planificar el desarrollo de la TDT local en Euskadi, ya que tuvo especial consideración hacia "la viabilidad de los proyectos: debían ser capaces de migrar solos y de cumplir las condiciones de la concesión sin necesidad de ayudas públicas" (Guimerà i Orts, 2011: 24).

Por otro lado, no todas las concesiones han sido otorgadas en demarcaciones en las que las televisiones locales operan en el año 2007. Ese es el caso de Televitoria y Teledonostia, las dos propiedad del Grupo Nervión ${ }^{3}$ y asociadas a Local Media. La pri-

${ }^{3}$ Grupo de comunicación vasco que cuenta con emisoras radiofónicas en las tres capitales de provincia de la Comunidad Autónoma -Radio Nervión, en Bilbao; Radio Donostia, en San Sebastián, y Radio Gorbea, en Vitoria-, así como con las emisoras de televisión local Telebilbao, Teledonostia y Televitoria. El grupo es el germen de la asociación de televisiones locales Local Media TV, que surge en 1994 como un proyecto basado en la afiliación de televisiones locales independientes. 
mera de ellas, en activo desde 1995 en Vitoria, no obtiene licencia en esa demarcación, sino en la de Llodio, mientras que Teledonostia tampoco consigue un canal en Donostia, donde funciona desde 2002, sino en Irún. De este modo, la digitalización deja fuera de su demarcación habitual a emisoras que cuentan con una trayectoria de varios años para hacer hueco a otras que no cuentan con ninguna experiencia en el sector televisivo local, como es el caso de EUVE, European Virtual Engineering Fundazioa, que obtiene concesión en Vitoria, y el Ayuntamiento de Donostia, que obtiene una concesión para la gestión directa de una canal digital en la demarcación de San Sebastián.

\subsection{Primeras consecuencias de la digitalización}

A pesar de que el Ejecutivo de Lakua se decanta por la adjudicación de licencias a proyectos audiovisuales consolidados, la digitalización y la crisis económica han supuesto un binomio difícil de soportar por el sector televisivo local en Euskadi. Una de sus consecuencias ha sido el desinterés por continuar en el sector demostrado por los grupos de comunicación de ámbito nacional presentes en el mismo desde comienzos de 2000, y que le han reportado a la televisión local unas estructuras empresariales con mayores posibilidades para negociar la publicidad local y para producir contenidos más atractivos (Román, 2005: 237).

Prisa fue el primero, en noviembre de 2008, en anunciar el cese de las emisiones de Localia TV en la Comunidad Autónoma Vasca a partir del 1 de enero de 2009. Sin embargo, las cuatro televisiones que desarrollan su actividad bajo esa marca en Euskadi, al estar participadas minoritariamente por el grupo, continúan son su actividad firmando un acuerdo con la empresa Contenalia, que se encarga de proveer a estas televisiones de los contenidos de producción ajena con los que completar sus parrillas.

Vocento es el siguiente grupo que, a partir de julio de 2009, comienza con el proceso de cese de las emisiones propias en las empresas en las que participa. Las dos primeras en sufrir la decisión de grupo son KTB-KateBerria, en Eibar (Gipuzkoa), en activo desde 1994, y Álava 7 TV, en Vitoria-Gasteiz, en activo desde 2002. Tras el encendido digital, el grupo continúa con esa misma estrategia. Así, en julio de 2010 pone fin a la actividad propia de Durango Telebista, que opera en Durango (Bizkaia) desde 2002. Poco más de un año después, en octubre de 2011, Vocento finaliza la actividad televisiva local de la emisora de la capital vizcaína, Bizkaia TV, que comienza sus emisiones en el año 2000 auspiciada por el Grupo Correo bajo el nombre de Bilbovisión.

Más reciente ha sido el cese de las antiguas emisoras Localia: Canal Gasteiz, localizada en Vitoria, concluye su actividad en febrero de 2012, mientras que en julio lo hacen Canal Bizkaia, situada en Bilbao; Gipuzkoa Telebista, en Donostia, y Txingudi Telebista, en Irún.

Los últimos meses también nos han dejado el cierre de la televisión local pionera en Euskadi, Telesko, en Eskoriatza (Gipuzkoa), que inicia su actividad siguiendo los pasos de las denominadas televisiones locales públicas de servicio a la comunidad (Navarro, 1999:72-78), financiada íntegramente por el Ayuntamiento y por las cuotas de los abonados al servicio. En activo desde 1985, la emisora, al transmitirse únicamente por cable, no concurrió al concurso de licencias digitales del Gobierno de Lakua. No obstante, la crisis económica y los recortes aplicados a las administracio- 
nes públicas han provocado que la emisora no reanudara su actividad en septiembre de 2012, concluido el periodo de descanso vacacional, tras decidir el Consistorio local el cese "temporal" de su emisión.

\section{Panorama actual de la televisión local vasca}

En septiembre de 2012, son 19 las televisiones locales que desarrollan su actividad en la Comunidad Autónoma Vasca con emisiones propias, seis menos que en 2007, cuando el Ejecutivo de Lakua procede al reparto de licencias digitales, lo supone un descenso del $24 \%$ frente a las posibilidades de crecimiento del $60 \%$ que estableció el Plan Técnico Nacional en este territorio. De entre las que continúan operando, la mayoría, un $47 \%$, se concentran en las tres capitales de provincia, con poblaciones superiores a los 100.000 habitantes; a continuación, se sitúan aquellas ubicadas en localidades de menos de 20.000 habitantes (32\%). De este modo, su distribución física se mantiene sin cambios significativos con respecto a la que presentaban en 2007.

Gráfico 1. Distribución de la televisión local en Euskadi (2012) (Elaboración propia)

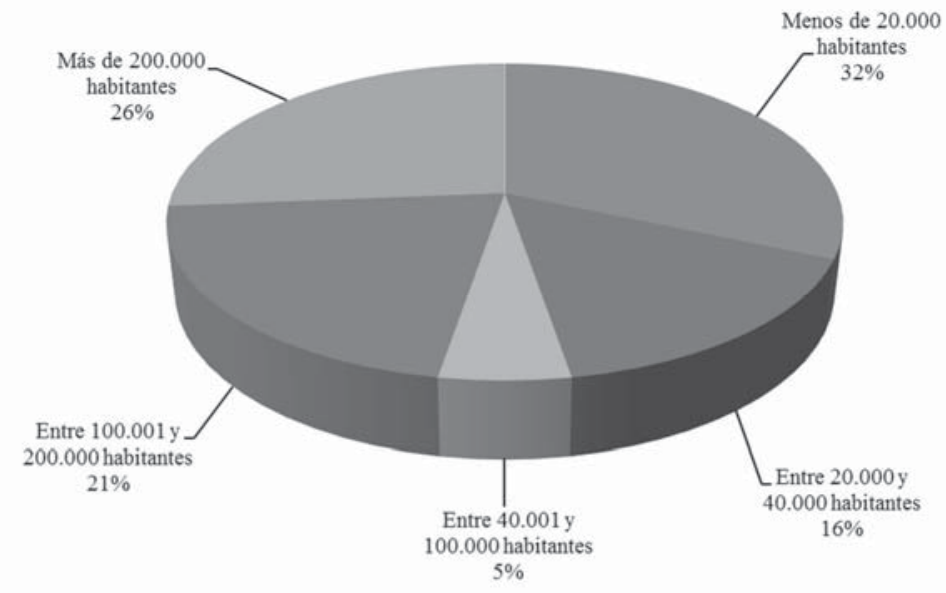

Por provincias, las siete demarcaciones establecidas en Gipuzkoa por el Plan Técnico Nacional posibilitan la convivencia de 28 canales digitales. No obstante, más de dos años y medio después del apagón analógico, son 11 las emisoras en activo, 3 menos que en 2007. En Araba, de los 8 posibles canales digitales tan sólo opera 1 frente a los 3 existentes en la última etapa analógica, mientras que en Bizkaia, las 24 televisiones locales teóricamente viables se han quedado en 7 , una menos que cuando el Gobierno Vasco procede al reparto de licencias digitales.

Una de las características más destacadas de estas emisoras es que prácticamente la mitad, un 47\%, son emisoras que cuentan con una importante tradición dentro del sector audiovisual vasco, ya que comenzaron a funcionar en la década de los 90 . Junto a éstas, están las que comenzaron su andadura entre los años 2000 y $2006(31,2 \%)$, mientras que el $15,8 \%$ corresponde a experiencias que han pasado a formar parte del panorama con posterioridad al reparto de licencias. 
Por otro lado, el desinterés mostrado por los grandes grupos de comunicación por estar presentes en el sector también se deja notar de forma significativa. Y es que mientras en el año 2007 eran un $72 \%$ las emisoras que estaban vinculadas a grupos de comunicación y asociaciones de televisión -Vocento (20\%), Prisa (16\%), Hamaika Telebista Hedatzeko Taldea (20\%) y Local Media (16\%)-, cinco años después, aunque este porcentaje no ha experimentado una variación significativa, situándose en el $69 \%$, sí que presenta diferencias en cuanto a su reparto. En este sentido, el mayor número de televisiones locales, el $64 \%$, está en manos de grupos de comunicación vascos, como son Hamaika Telebista Hedatzeko Taldea, único que ha puesto en marcha nuevas emisoras tras la adjudicación de licencias digitales, y la asociación Local Media, que cuenta con los canales propiedad del Grupo Nervión en las tres capitales de provincia, a las que se suman dos asociadas más en Gipuzkoa, agrupando al 27\% de total.

Gráfico 2. Titularidad de la televisión local en la Comunidad Autónoma Vasca (2012) (Elaboración propia)

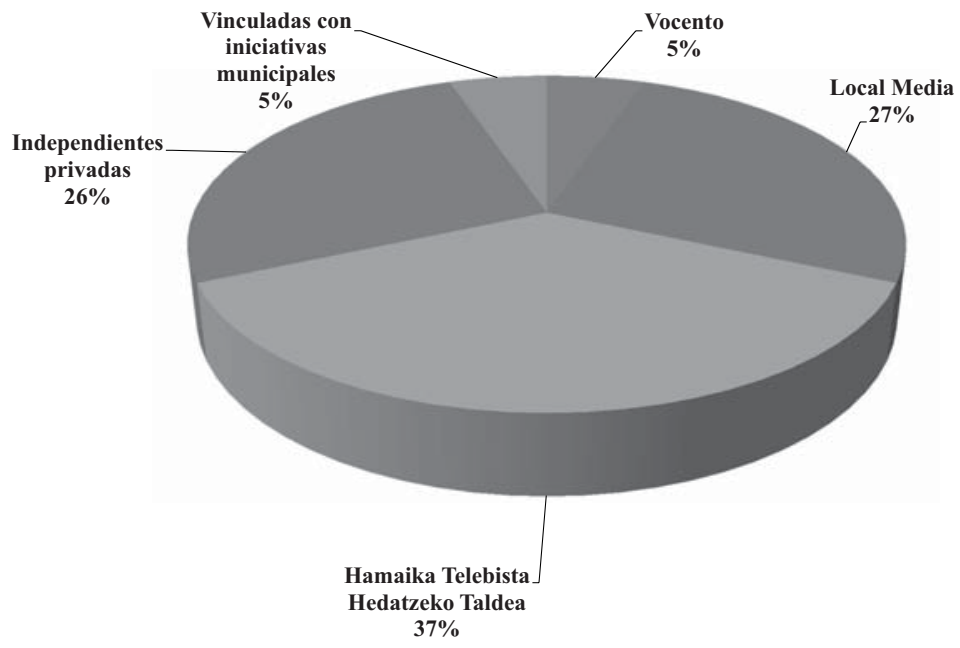

Entre las que no están vinculadas con ningún grupo de comunicación o asociación de televisión local, ha aumentado la presencia de aquellas que usan Internet como único medio para difundir su señal, mientras que las iniciativas públicas han disminuido su presencia tras el cierre de Telesko.

\section{La oportunidad de Internet}

Actualmente, prácticamente todas las televisiones locales en activo están presentes en Internet, una situación que contrasta con la existente en 2007, donde tan sólo un $48 \%$ las televisiones estaban en la Red -un 40\% disponía de página web y el $8 \%$ empleaba Internet como sistema de transmisión-, mientras que el 32\% no disponía de página web y el 20\% estaba en construcción (Gómez, 2010: 454).

$\mathrm{Si}$ "una de las ventajas que ofrece el tener una página web por parte de una televisión local es que puede ser tan buena a todos los niveles (diseño, velocidad, contenidos, 
información, claridad, etc.) como la de una televisión generalista, que maneja unos presupuestos muy superiores" (Morales, 2008), en la era digital Internet se ha convertido también en el medio que permite la continuidad de emisoras locales que, por motivos económicos, no han podido participar del proceso digital terrestre. Así, ese $8 \%$ de televisiones que en 2007 desarrollaban su actividad como televisiones locales transmitiéndose únicamente a través de la Red, actualmente se ha incrementado hasta el 21\%.

Entre ellas, se encuentran las veteranas Proyección TV, Euskaraz TV y Altza TV, a las que se han unido Ibaizabal Telebista, que comenzó su actividad en el año 2008, con el objetivo de reflejar la situación lingüística y comunicativa de Bilbao, y Urnieta TB, que tras 18 años informando sobre lo acontecido en su entorno más próximo, en julio de 2010 pasa a emitir sus contenidos audiovisuales en la Red, a través de un enlace habilitado en la página web del Ayuntamiento de Urnieta (Gómez, 2011: 151). Responsables de la emisora señalan que el cambio a la emisión por Internet ha sido obligado, puesto que con la llegada del apagón analógico, ésta se veía abocada a dejar de emitir. De este modo, la Red "supone una oportunidad para que sobreviva la televisión local", ya que "permite mantener un canal de televisión sin estar obligado a ofrecer contenido continuo la mayoría de las horas del día y sin la estructura ni los costes a los que obligan las emisiones habituales" (Roger, 2012: web). Además, a pesar de que Internet se configura "como un medio de difusión que rebasa fronteras y elimina las distancias", también ofrece "múltiples recursos para establecer comunidad entre los usuarios, lo que favorece el sentido de pertenencia a un ámbito concreto, como es el de las televisiones locales" (Mendiguren, 2010: 112).

\section{Conclusiones}

La digitalización, unida a la crisis económica y publicitaria que está sufriendo el mercado audiovisual en general, ha llevado al cese en la Comunidad Autónoma Vasca de televisiones locales con una importante trayectoria en el sector. El optimismo inicial, que permitía la convivencia de un total de 60 canales digitales locales en Euskadi -28 de Gipuzkoa, 24 en Bizkaia y 8 en Araba- se ha traducido, dos años y medio después del encendido digital, en un descenso cuantitativo con respecto a la realidad del sector en 2007. De hecho, un $24 \%$ de los canales que operaban cuando el Gobierno Vasco procedió al reparto de licencias digitales han cesado sus emisiones propias desde el año 2009.

Aunque los grupos de comunicación y asociaciones de televisión local fueron los grandes beneficiarios del reparto de licencias digitales, acaparando más del $90 \%$ de las concesiones, aquellos de ámbito estatal presentes en el sector desde comienzos del tercer milenio han desaparecido, como ha sido el caso de Prisa (Localia TV), o han pasado a contar con una presencia minoritaria, como es el caso de Vocento: de dominar el 20\% de la televisión local en el año 2007, actualmente tan sólo uno de sus canales mantiene sus emisiones propias en la capital guipuzcoana. Esto hace que el sector haya pasado a estar en manos de iniciativas vinculadas al grupo vasco Hamaika Telebista Hedatzeko Taldea (37\%) y Local Media (27\%), liderado en Euskadi por el Grupo Nervión, al tiempo que las experiencias privadas independientes (26\%) también cuentan con una presencia destacada, fundamentalmente de la mano de aquellas que realizan sus emisiones a través de Internet, que han pasado del 8 al 21\%. 
Por lo demás, la televisión local en la era digital continúa siendo una televisión que prefiere las capitales de provincia y los municipios con menos de 20.000 habitantes para realizar sus emisiones.

\section{Referencias bibliográficas}

BADILLO, Ángel y ORTEGA, Félix (2008): "La nueva televisión digital local en España: los grupos en el audiovisual de proximidad", en: http://www.ae-ic.org/santiago2008/contents/pdf/comunicaciones/267.pdf [fecha de consulta: 10 de noviembre de 2010].

GARCÍA. Joaquín (2005): "Posibilidades y retos de la televisión digital", en Razón y Palabra, $\mathrm{n}^{\circ}$ 45: http://www.razonypalabra.org.mx/anteriores/n45/jgarcia.html [fecha de consulta: el 23 de marzo de 2010).

GÓMEZ RUBIO, Leire (2011): "En la encrucijada digital. Un modelo de proximidad en apuros: la televisión local en Euskadi tras el apagón analógico", en ALCUDIA BORREGUERO, M. et al (Coor.): Competidores y aliados. Medios en convergencia, los nuevos retos en comunicación. Madrid, CEU Ediciones, pp. 143-160.

GÓMEZ RUBIO, Leire (2010): Análisis prospectivo de un modelo de proximidad: la televisión local en la Comunidad Autónoma Vasca ante la llegada de la TDT. Tesis Doctoral. Universidad del País Vasco-Euskal Herriko Unibertsitatea.

GUIMERÀ i ORTS, Josep Àngel (2011): "Políticas de TDT y configuración del mapa televisivo autonómico digital en la Comunidad Autónoma Vasca", en Revista Zer, vol. $16, \mathrm{n}^{\circ} 3$, pp. 13-30.

MENDIGUREN GALDOSPIN, Terese (2010): “Análisis comparativo de las páginas web de las televisiones locales de la comarca del Gran Bilbao", en Revista ZER, vol. $15, \mathrm{n}^{\circ} 29$, pp. 89-114.

MONEDERO MORALES, Rocío del Carmen (2009): "TDT.com: el futuro de la televisión local pasa por la web", en ponencia presentada en el XII Congreso Iberoamerica de Internet, Telecomunicaciones y Sociedad de la Información (Mundo Internet): http://www.mundointernet.es/IMG/pdf/ponencia187.pdf [fecha de consulta: 14 de marzo de 2012)

NAVARRO MORENO, José Antonio (1999): La televisión local. Andalucía: la nueva comunicación. Madrid, Fragua.

ROGER, Maiol (2012): "Internet, refugio de la televisión loca", en EL PAÍS, 22 de enero: http://ccaa.elpais.com/ccaa/2012/01/22/catalunya/1327263174_295861 .html [fecha de consulta: 24 de junio de 2012]

ROMÁN PORTAS, Mercedes (2005): “La televisión local en España”, en Sphera Pública, $\mathrm{n}^{\mathrm{0}} 5$, pp. 229-239.

\section{Leire GÓMEZ RUBIO}

Doctora en Periodismo por la Universidad del País Vasco (UPV/EHU)

leiregom@yahoo.es 